\title{
Semileptonic Hyperon Decays and CKM Unitarity*
}

\author{
Nicola Cabibbo \\ Department of Physics, University of Rome - La Sapienza \\ and INFN, Sezione di Roma 1 \\ Piazzale A. Moro 5, 00185 Rome, Italy \\ nicola.cabibbo@roma1.infn.it \\ Earl C. Swallow \\ Department of Physics, Elmhurst College \\ Elmhurst, Illinois 60126 and Enrico Fermi Institute, \\ The University of Chicago, Chicago, Illinois \\ earls@elmhurst.edu \\ Roland Winston \\ Division of Natural Sciences, \\ The University of California - Merced, \\ Merced, California 95344 \\ rwinston@ucmerced.edu \\ (Dated: October 26, 2018)
}

\begin{abstract}
Using a technique that is not subject to first-order $S U(3)$ symmetry breaking effects, we determine the $V_{u s}$ element of the CKM matrix from data on semileptonic hyperon decays. We obtain $V_{u s}=0.2250(27)$, where the quoted uncertainty is purely experimental. This value is of similar experimental precision to the one derived from $K_{l 3}$, but it is higher and thus in better agreement with the unitarity requirement, $\left|V_{u d}\right|^{2}+\left|V_{u s}\right|^{2}+\left|V_{u b}\right|^{2}=1$. An overall fit including the axial contributions, and neglecting $S U(3)$ breaking corrections, yields $F+D=1.2670 \pm 0.0035$ and $F-D=-0.341 \pm 0.016$ with $\chi^{2}=2.96 / 3$ d.f.

PACS numbers: 12.15.Hh, 13.30.Ce, 14.20.Jn
\end{abstract}

The determination of the elements of the CabibboKobayashi-Maskawa (CKM) matrix [1, 2] is one of the main ingredients for evaluating the solidity of the standard model of elementary particles. This is a vast subject which has seen important progress with the determination 3, 4] of $\epsilon^{\prime} / \epsilon$ and the observation 5, 6] of $C P$ violation in $\mathrm{B}$ decays.

While a lot of attention has recently been justly devoted to the higher mass sector of the CKM matrix, it is the low mass sector, in particular $V_{u d}$ and $V_{u s}$ where the highest precision can be attained. The most sensitive test of the unitarity of the CKM matrix is provided by the relation $\left|V_{u d}\right|^{2}+\left|V_{u s}\right|^{2}+\left|V_{u b}\right|^{2}=1-\Delta$. Clearly the unitarity condition is $\Delta=0$. The $\left|V_{u b}\right|^{2}$ contribution 7] is negligible $\left(10^{-5}\right)$ at the current level of precision. The value $V_{u d}=0.9740 \pm 0.0005$ is obtained from superallowed pure Fermi nuclear decays 8]. In combination with $V_{u s}=0.2196 \pm 0.0023$, derived from $K_{e 3}$ decay 9, 10], this yields $\Delta=0.0032 \pm 0.0014$. On its face, this represents a 2.3 standard deviation departure from unitarity [8].

In this communication we reconsider the contribution that the hyperon beta decays can give to the determination of $V_{u s}$. The conventional analysis of hyperon beta decay in terms of the parameters $F, D$ and $V_{u s}$ is marred

${ }^{*}$ To be published in Physical Review Letters. by the expectation of first order $S U(3)$ breaking effects in the axial-vector contribution. The situation is only made worse if one introduces adjustable $S U(3)$ breaking parameters as this increases the number of degrees of freedom and degrades the precision. If on the contrary, as we do here, one focuses the analysis on the vector form factors, treating the rates and $g_{1} / f_{1}$ [11] as the basic experimental data, one has directly access to the $f_{1}$ form factor for each decay, and this in turn allows for a redundant determination of $V_{u s}$. The consistency of the values of $V_{u s}$ determined from the different decays is a first confirmation of the overall consistency of the model. A more detailed version of this work will be published in the Annual Reviews of Nuclear and Particle Sciences 12.

In 1964 Ademollo and Gatto proved [13] that there is no first-order correction to the vector form factor, $\Delta^{1} f_{1}(0)=0$. This is an important result: since experiments can measure $V_{u s} f_{1}(0)$, knowing the value of $f_{1}(0)$ in $\Delta S=1$ decays is essential for determining $V_{u s}$.

The Ademollo-Gatto Theorem suggests an analytic approach to the available data that first examines the vector form factor $f_{1}$ because it is not subject to first-order $S U(3)$ symmetry breaking effects. An elegant way to do this is to use the measured value of $g_{1} / f_{1}$ along with the predicted values of $f_{1}$ and $f_{2}$ to extract a $V_{u s}$ value from the decay rate for each decay. If the theory is correct, these should coincide within errors, and can be combined to obtain a best value of $V_{u s}$. This consistency of the $V_{u s}$ 
values obtained from different decays then indicates the success of the Cabibbo model. A similar approach appears to have been taken in Ref. 14].

Four hyperon beta decays have sufficient data to perform this analysis: $\Lambda \rightarrow p e^{-} \bar{\nu}, \Sigma^{-} \rightarrow n e^{-} \bar{\nu}, \Xi^{-} \rightarrow$ $\Lambda e^{-} \bar{\nu}, \quad \Xi^{0} \rightarrow \Sigma^{+} e^{-} \bar{\nu}[9]$. Table \ shows the results for them. In this analysis, both model-independent and model-dependent radiative corrections [1] are applied and $q^{2}$ variation of $f_{1}$ and $g_{1}$ is included. Also $S U(3)$ values of $g_{2}=0$ and $f_{2}$ are used along with the numerical rate expressions tabulated in Ref. 11]. We have not however included $S U(3)$-breaking corrections to the $\mathrm{f}_{1}$ form factor, which will be discussed in the next section. The stated $V_{u s}$ errors are purely experimental, coming from experimental uncertainties in the hyperon lifetimes, branching ratios, and form factor ratios.

The four values are clearly consistent $\left(\chi^{2}=2.26 / 3\right.$ d.f. $)$ with the combined value of $V_{u s}=0.2250 \pm 0.0027$. This value is nearly as precise as that obtained from kaon decay $\left(V_{u s}=0.2196 \pm 0.0023\right)$ and, as observed in previous analyses 15, 16, 26], is somewhat larger. In combination with $V_{u d}=0.9740 \pm 0.0005$ obtained from superallowed pure Fermi nuclear decays [8], the larger $V_{u s}$ value from hyperon decays beautifully satisfies the unitarity constraint $\left|V_{u d}\right|^{2}+\left|V_{u s}\right|^{2}+\left|V_{u b}\right|^{2}=1$.

TABLE I: Results from $V_{u s}$ analysis using measured $g_{1} / f_{1}$ values

\begin{tabular}{lccc}
\hline \hline $\begin{array}{l}\text { Decay } \\
\text { Process }\end{array}$ & $\begin{array}{c}\text { Rate } \\
\left(\mu \mathrm{sec}^{-1}\right)\end{array}$ & $g_{1} / f_{1}$ & $V_{u s}$ \\
\hline$\Lambda \rightarrow p e^{-} \bar{\nu}$ & $3.161(58)$ & $0.718(15)$ & $0.2224 \pm 0.0034$ \\
$\Sigma^{-} \rightarrow n e^{-} \bar{\nu}$ & $6.88(24)$ & $-0.340(17)$ & $0.2282 \pm 0.0049$ \\
$\Xi^{-} \rightarrow \Lambda e^{-} \bar{\nu}$ & $3.44(19)$ & $0.25(5)$ & $0.2367 \pm 0.0099$ \\
$\Xi^{0} \rightarrow \Sigma^{+} e^{-} \bar{\nu}$ & $0.876(71)$ & $1.32(+.22 /-.18)$ & $0.209 \pm 0.027$ \\
Combined & - & - & $0.2250 \pm 0.0027$ \\
\hline
\end{tabular}

We will limit our discussion to the effects that are most relevant for the determination of $V_{u s}$. Turning our attention first to $S U(3)$-breaking corrections to the $f_{1}$ form factor, we find in the literature computations that use some version of the quark model, as in [17, 18], or some version of chiral perturbation theory, as in [15, 19, 20].

The quark-model computations find that the $f_{1}$ form factors for the different $\Delta S=1$ decays are reduced by a factor, the same for all decays, given as 0.987 in [17, and 0.975 in [18], a decrease respectively of $1.3 \%$ or $2.5 \%$. This is a very reasonable result, the decrease arising from the mismatch of the wave functions of baryons containing different numbers of the heavier $s$ quarks.

Evaluations of $f_{1}$ in chiral perturbation theory range from small negative corrections in [19] to larger positive corrections in [15, 20]. Positive corrections in $f_{1}$ for all hyperon beta decays cannot be excluded, but are certainly not expected in view of an argument 21] according to which one expects a negative correction to $f_{1}$ at least in the $\Sigma^{-} \rightarrow n e^{-} \bar{\nu}$ case. This result follows from the observation that the intermediate states that con- tribute to the positive second-order terms in the Ademollo and Gatto sum rule have, in this case, quantum numbers $S=-2, I=3 / 2$; no resonant baryonic state is known with these quantum numbers. If we accept the hypothesis that the contribution of resonant hadronic states dominate, we can conclude that the correction to $f_{1}$ in $\Sigma^{-}$beta decay should be negative. We note that this argument also applies to $K_{l 3}$ decays, and that the corrections to these decays, computed with chiral perturbation theory, are, as expected, negative.

A modern revisitation of the quark-model computations will be feasible in the near future with the technologies of lattice QCD, and we would expect that a small negative correction would be obtained in quenched lattice QCD, an approximation that consists in neglecting components in the wave function of the baryons with extra quark-antiquark pairs. This is known to be an excellent approximation in low-energy hadron phenomenology [22].

Multiquark effects can be included in lattice QCD by forsaking the quenched approximation for a full simulation. Alternatively one could resort to chiral perturbation theory to capture the major part of the multiquark contributions which will be dominated by virtual $\pi, K, \eta$ states. Early results of a similar strategy applied to the $K_{e 3}$ decays 23] indicate that in that case a $1 \%$ determination of the $f_{+}(0)$ form factor is within reach, and we expect that a similar precision can be obtained in the case of hyperon decays. In the present situation we consider it best not to include any $S U(3)$ breaking corrections in our evaluation, nor to include an evaluation of a theoretical error. Our expectation that the corrections to $f_{+}(0)$ will be small and negative can only be substantiated by further work.

We next turn our attention to the possible effect of ignoring the $g_{2}$ form factor. In the absence of second class currents 24] the form factor $g_{2}$ can be seen to vanish in the $S U(3)$ symmetry limit. The argument is very straightforward: the neutral currents $A_{\alpha}^{3}=\bar{q} \lambda^{3} \gamma_{\alpha} \gamma_{5} q$ and $A_{\alpha}^{8}=\bar{q} \lambda^{8} \gamma_{\alpha} \gamma_{5} q$ that belong to the same octet as the weak axial current are even under charge conjugation, so that their matrix elements cannot contain a weak - electricity term, which is $C$-odd. The vanishing of the weak electricity in the proton and neutron matrix elements of $A_{\alpha}^{3}, A_{\alpha}^{8}$ implies the vanishing of the $D$ and $F$ coefficients for $g_{2}(0)$, so that, in the $S U(3)$ limit, the $g_{2}(0)$ form factor vanishes for any current in the octet.

In hyperon decays a nonvanishing $g_{2}(0)$ form factor can arise from the breaking of $S U(3)$ symmetry. Theoretical estimates [25] indicate a value for $g_{2}(0) / g_{1}(0)$ in the -0.2 to -0.5 range.

In determining the axial-vector form factor $g_{1}$ from the Dalitz Plot - or, equivalently, the electron-neutrino correlation - one is actually measuring $\tilde{g}_{1}$, a linear combination of $g_{1}$ and $g_{2}\left(\tilde{g}_{1} \approx g_{1}-\delta g_{2}\right.$ up to first order in $\delta=\Delta M / M)$. This has already been noticed in past experiments and is well summarized in Gaillard and Sauvage [26], Table 8. Therefore, in deriving $V_{u s}^{2} f_{1}^{2}$ (hence $V_{u s}$ ) from the beta decay rate, there is in fact a 
small sensitivity to $g_{2}$. To first order, the rate is proportional to $V_{u s}^{2}\left[f_{1}^{2}+3 g_{1}^{2}-4 \delta g_{1} g_{2}\right] \approx V_{u s}^{2}\left[f_{1}^{2}+3 \tilde{g}_{1}^{2}+2 \delta \tilde{g}_{1} g_{2}\right]$. In fact, this is a second order correction to the value of $V_{u s}$, potentially of the same order of magnitude as the corrections to $f_{1}$.

Experiments that measure correlations with polarization - in addition to the electron-neutrino correlation are sensitive to $g_{2}$. While the data are not yet sufficiently precise to yield good quantitative information, one may nevertheless look for trends. In polarized $\Sigma^{-} \rightarrow p e^{-} \bar{\nu}$ [27], negative values of $g_{2} / f_{1}$ are clearly disfavored (a positive value is preferred by $1.5 \sigma$ ). Since the same experiment unambiguously established that $g_{1} / f_{1}$ is negative one concludes that allowing for nonvanishing $g_{2}$ would increase the derived value of $V_{u s}^{2} f_{1}^{2}$. In polarized $\Lambda \rightarrow p e^{-} \bar{\nu}$ the data favor 28] negative values of $g_{2} / f_{1}$ (by about $2 \sigma$ ). In this decay, $g_{1} / f_{1}$ is positive so that again, allowing for the presence of nonvanishing $g_{2}$ would increase the derived value of $V_{u s}^{2} f_{1}^{2}$. In either case, we may conclude that making the conventional assumption of neglecting the $g_{2}$ form factor tends to underestimate the derived value of $V_{u s}$. A more quantitative conclusion must await more precise experiments. We consider it to be of the highest priority to determine the $g_{2}$ form-factor (or a stringent limit on its value) in at least one of the hyperon decays, ideally in $\Lambda$ semileptonic decay which at the moment seems to offer the single most precise determination of $V_{u s}$.

The excellent agreement with the unitarity condition of our determination of $V_{u s}$, which neglects $S U(3)$-breaking effects, seems to indicate that such effects were overestimated in the past, probably as a consequence of the uncertainties of the early experimental results. We also find 12. that the $g_{1}$ form factor of the different decays, which is subject to first order corrections, is well fitted by the $F, D$ parameters [1], with $F+D=1.2670 \pm 0.0035$ and $F-D=-0.341 \pm 0.016$ with $\chi^{2}=2.96 / 3$ d.f.

The value of $V_{u s}$ obtained from hyperon decays is of comparable experimental precision with that obtained from $K_{l 3}$ decays, and is in better agreement with the value of $\theta_{C}$ obtained from nuclear beta decay. While a discrepancy between $V_{u s}$ and $V_{u d}$ could be seen as a portent of exciting new physics, a discrepancy between the two different determinations of $V_{u s}$ can only be taken as an indication that more work remains to be done both on the theoretical and the experimental side.

On the theoretical side, renewed efforts are needed for the determination of $S U(3)$-breaking effects in hyperon beta decays as well as in $K_{l 3}$ decays. While it is quite possible to improve the present situation on the quark-model front, the best hopes lie in lattice QCD simulations, perhaps combined with chiral perturbation theory for the evaluation of large-distance multiquark contributions.

We have given some indication that the trouble could arise from the $K_{l 3}$ determination of $V_{u s}$, and we would like to encourage further experimental work in this field [29]. We are however convinced of the importance of renewed experimental work on hyperon decays, of the kind now in progress at the CERN SPS. The interest of this work goes beyond the determination of $V_{u s}$, as it involves the intricate and elegant relationships that the model predicts.

The continuing intellectual stimulation provided by colleagues in the Fermilab KTeV Collaboration, particularly members of the hyperon working group, is gratefully acknowledged. This work was supported in part by the U.S. Department of Energy under grant DE-FG0290ER40560 (Task B).
[1] N. Cabibbo, Phys. Rev. Lett. 10, 531 (1963)

[2] M. Kobayashi and T. Maskawa, Prog. Theor. Phys. 49, 652 (1973).

[3] KTeV Collaboration, A. Alavi-Harati et al., Phys. Rev. D 67:012005 (2003)

[4] NA48 Collaboration, A. Lai et al., Eur. Phys. J. C 22, 231 (2001) arXiv:hep-ex/0110019.

[5] BABAR Collaboration, B. Aubert et al., Phys. Rev. Lett. 87, 091801 (2001).

[6] Belle Collaboration, K. Abe et al., Phys. Rev. Lett. 87, 091802 (2001).

[7] BABAR Collaboration, B. Aubert et al., Phys. Rev. Lett. 90, 181801 (2003).

[8] I. S. Towner and J. C. Hardy, Santa Fe 1998, Physics beyond the standard model, 338-359; arXiv:nucl-th/9809087.

[9] K. Hagiwara et al. (Particle Data Group), Phys. Rev. D 66:010001 (2002)

[10] H. Leutwyler and M. Roos, Z. Phys. C 25, 91 (1984)

[11] A. Garcia and P. Kielanowski, The Beta Decay of Hyperons, Lecture Notes in Physics 222, Springer-Verlag, Berlin, 1985. With the metric and $\gamma$ matrix conventions used here, $g_{1} / f_{1}$ is positive for neutron beta decay.

[12] N. Cabibbo, E. C. Swallow, R Winston, Ann. Rev. Nucl. Part. Sci. Dec. 2003, 53: 39-75.

[13] M. Ademollo and R. Gatto, Phys. Rev. Lett. 13, 264 (1964).

[14] P. M. Gensini and G. Violini, hep-ph/9311270

[15] R. Flores-Mendieta, E. Jenkins and A. V. Manohar, "SU(3) symmetry breaking in hyperon semileptonic decays," Phys. Rev. D 58, 094028 (1998) arXiv:hep-ph/9805416.

[16] R. Flores-Mendieta, A. Garcia, G. Sanchez-Colon Phys. Rev. D54:6855 (1996);

[17] J. F. Donoghue, B. R. Holstein and S. W. Klimt, "K-M Angles And SU(3) Breaking In Hyperon Beta Decay," Phys. Rev. D 35, 934 (1987).

[18] F. Schlumpf, "Beta decay of hyperons in a relativistic quark model," Phys. Rev. D 51, 2262 (1995) arXiv:hep-ph/9409272.

[19] A. Krause, "Baryon Matrix Elements Of The Vector Current In Chiral Perturbation Theory," Helv. Phys. Acta 63, 3 (1990).

[20] J. Anderson and M. A. Luty, "Chiral corrections to hy- 
peron vector form-factors," Phys. Rev. D 47, 4975 (1993) arXiv:hep-ph/9301219.

[21] H. R. Quinn and J. D. Bjorken, "Renormalization Of Weak Form-Factors, And The Cabibbo Angle," Phys. Rev. 171, 1660 (1968).

[22] J. Sexton, D. Weingarten, Phys. Rev. D55:4025 (1997)

[23] We are grateful to Guido Martinelli for a discussion on this point, see D. Bec̀irevic̀ et al., arXiv:hep-ph/0403217.

[24] S. Weinberg, Phys. Rev. 112, 1375 (1958).

[25] Barry R. Holstein, "Hyperon 99", Proceedings of the Hyperon Physics Symposium, pgs. 4-9, Fermilab, September
27-29 1999, D.A. Jensen, E. Monnier, Editors.

[26] J. M. Gaillard and G. Sauvage, Ann. Rev. Nucl. Part. Sci. 34, 351 (1984).

[27] S. Y. Hsueh, et al., Phys. Rev. D 38, 2056 (1988).

[28] R. Oehme, R. Winston, A. Garcia, Phys. Rev. D 3, 1618 (1971).

[29] In fact, a recently reported result from the Brookhave E865 Collaboration indicates a higher $K_{e 3}$ decay rate than that previously used to determine $V_{u s}$. See A. Sher, et al., hep-ex/0305042 (June 24, 2003). 